\title{
A Research Paper on Internet of Things based upon Smart Homes with Security Risk Assessment using OCTAVE Allegro
}

\author{
Ahmad Bilal Zia ${ }^{1}$ \\ ${ }^{l}$ M.Tech(CSE), \\ School of Technology \& Sciences, \\ Alakh Prakash Goyal Shimla University, Shimla (HP ),
}

\author{
Ms. Kshamta Chauhan ${ }^{2}$ \\ ${ }^{2}$ Assistant Professor, \\ School of Technology \& Sciences, Alakh Prakash Goyal \\ Shimla University, Shimla (HP),
}

Abstract - The new and problematic age of shrewd residential bundles (from this point forward called applications) in light of Internet of Things (IoT) is essentially controlled and dispersed. To give significant bits of knowledge into mechanical situations and bolster analysts, we should perceive the to be had choices and holes in this line of exploration. Along these lines, on this glance at, an outline is done to delineate examinations scene directly into a sound scientific classification. In this paper, I have a pattern to audit a few deals with savvy homes utilizing web o things (IOT) as of late. We have pattern to examine numerous ways use for steps of brilliant homes like keen home security, savvy home condition, shrewd home gadgets and association. Web of Things (IoT) is a rising development that is making our world increasingly savvy. Associated world can't be imagined without IoT. An IoT based Smart Home is one such model. In IoT engaged Smart Home condition various things, for instance, lighting, home contraptions, PCs, observation camera, etc all are related with the Internet and empowering customer to screen and control things offering little appreciation to time and region necessity. This paper delineates Frugal Labs IoT Platform (FLIP) for building IoT enabled Smart Home. This paper looks at components of Smart Home and its applications and presents FLIP plan with execution of Smart Home organizations using FLIP through a proposed structure. The proposed system showed in this paper is used for checking and controlling Smart Home condition. Web of Things (IoT) conceptualizes the chance of remotely partner and watching genuine (things) through the Internet. Concerning our home, this thought can be suitably combined to make it progressively astute, increasingly secure and robotized. This IoT adventure bases on building a sharp remote home security structure which sends alerts to the owner by using Internet if there ought to emerge an event of any trespass and raises an alarm on the other hand. Also, the comparable can in like manner be utilized for home automation by using a comparative course of action of sensors. One of the most widely recognized goal to distribute this paper to utilize sensors, security gadgets, security dangers and machine to machine correspondence in our savvy home frameworks, which depends on Internet of Things(IoT) and furthermore utilizing a sort of confirmation for making the brilliant focal controller secure.

Keywords - Internet of things (IOT), smart home, Security, device, controller, Machine to Machine Communication, Security Threats, Security Countermeasures.

\section{INTRODUCTION}

Internet has changed human's lifestyles by way of presenting anytime, anywhere connectivity with all and sundry. As many advancements in generation has been come the sensors, processors, transmitters, receivers, and so on. Are now available in very cheap price. Hence these all matters can be utilized in our daily lifestyles. If absolutely everyone desires to expand the services of net, then Internet of Things can be stated as the expansion of internet offerings. Today's net is now increasing towards Internet of Things (IoT). The idea of Internet of things(IoT) became presented with the aid of the improvement of the widely used international system known as the web along the company of ubiquitous computing and mobiles in eager articles which brings new open doorways for the creation of innovative answers for distinctive parts of existence. The idea of Internet of factors(IoT)creates a system of items which could convey, associate and collaborate collectively to reach a shared goal [1]. IoT gadgets can improve our ordinary lives, as each device stops performing as a solitary system and turn out to be a few part of an entire complete associated framework. This gives us with the following statistics to be broke down for better fundamental leadership, monitoring our agencies and looking at our homes whilst we are far far from them

Internet-of-Things: The internet wherein the existing network of internet to the pc structures will connect with the real global items or things. Things may include any items, domestic home equipment, gadgets, vehicles, and so forth. And while these things connect with the internet in particular infrastructure via general protocols then the whole machine is said to be Internet of Things (IoT). The Internet of Things (IoT) is a system of interrelated computing devices, mechanical and digital machines, gadgets, animals or humans that are furnished with precise identifiers (UIDs) and the capacity to transfer records over a network with out requiring human-to-human or human-to-pc interaction.

The definition of the Internet of Things has evolved because of the convergence of a couple of technology, actual-time analytics, system reading, and commodity sensors, and embedded structures. Traditional fields of embedded systems, wireless sensor networks, control systems, automation (inclusive of domestic and building automation), and others all contribute to allowing the Internet of Things. In the consumer market, IoT technology is most synonymous with merchandise regarding the concept of the "smart domestic", overlaying gadgets and home equipment (collectively with lighting, thermostats, domestic safety systems and cameras, and different domestic home 
equipment) that manual one or extra common ecosystems, and may be managed thru devices related to that environment, along side clever telephones and smart audio gadget.[2]

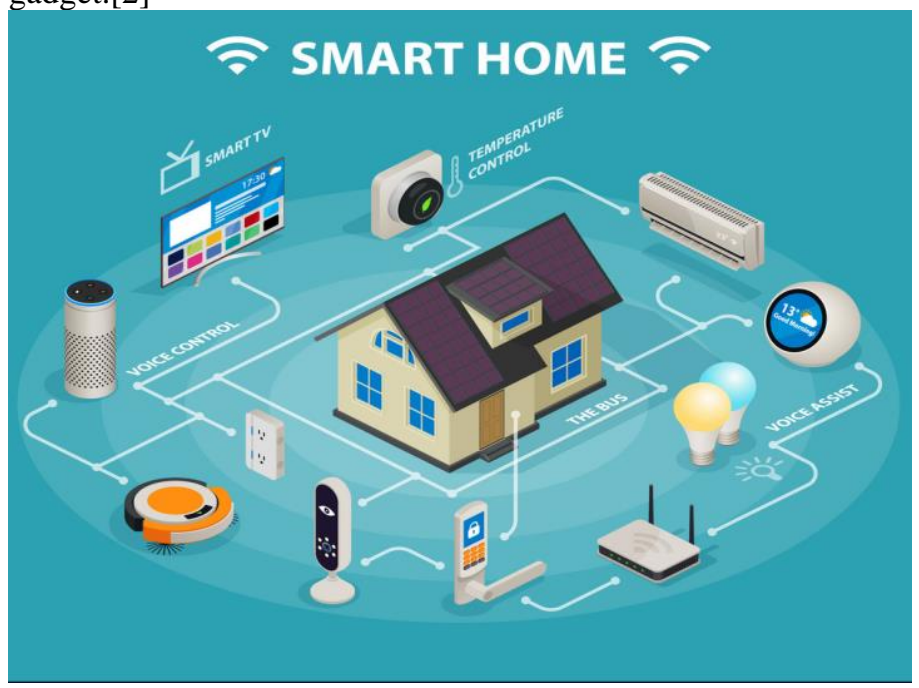

Fig 1: A Smart Home System using Internet of Things (IOT)

\section{INTERNET OF THINGS FOR SMART HOME}

IoT contraptions are a bit of the greater thought of home robotization, which can fuse lighting, warming and cooling, media and security systems. Long stretch preferences could join essentialness venture reserves by means of thusly ensuring lights and equipment are murdered. Endeavors are pondering and recognizing a huge proportion of IoT-related applications, which can be isolated into two classes. In first grouping the devices are related, molding an establishment that is mechanized with $\mathrm{M} 2 \mathrm{M}$ correspondence and significance to improve people's lives. In this grouping IoT can be seen expecting the activity of TCC\&R (track, request and control). In nuclear families for example the room temperature, windows, lights and electrical contraptions, etc would all have the option to be controlled remotely from PC and robotized to discard manual techniques people face step by step in their lives. The Internet of Things is acknowledged to have suffering effects in both advancement and present day society. In present day information society, IoT can be seen as an overall system that enables additionally created organizations by partner physical and virtual contraptions and things to starting at currently existing and regardless, best in class information and correspondence developments.

In spite of the way that the term IoT has started to incline out in the open during the latest five years, partner things to the Internet is definitely not another wonder. Possibly the key application was the Trojan room coffee pot that was envisioned not long after web was considered in 1989. A keen home or robotized home could be established on a phase or focus focuses that control clever devices and mechanical assemblies. For instance, using Apple's Home Kit, producers can have their home things and decoration obliged by an application in iOS devices, for instance, the iPhone and the Apple Watch. This could be a dedicated application or iOS neighborhood applications, for instance, Siri. This can be displayed by virtue of Lenovo's Smart Home Essentials, which is a line of keen home contraptions that are controlled through Apple's Home application or Siri without the necessity for a Wi-Fi interface. The general work in this paper and my examination is to make keen home utilizing web of things with a high security level and including a few gadgets, sensors, controller and so on.

\section{AIM and OBJECTIVE}

This exposition is an IoT programming assembled strategy as for the field of Home Automation. Customary use-cases fuse evaluating home conditions, controlling home mechanical assemblies and controlling home access through RFID cards for example and windows through servo locks [9]. In any case, the essential point of convergence of this paper is to grow the security of homes through IoT. Even more expressly, checking and controlling servo door locks, passage sensors, perception cameras, surveillance vehicle and smoke cautions, which help ensuring and boosting prosperity and security of homes. A client has the accompanying highlights through a versatile application where he/she:

1. can turn on or off LED lights and screen the condition of the LED.

2. can bolt and open entryways through servo engines and screen if the entryways are bolted or opened.

3. can screen if the entryways are shut or opened through IR sensors.

4. is advised through email if the entryway is left open for a really long time.

5. is advised of who entered through the entryway as the camera catches the face picture and send it to him/her by means of email.

6. is informed through email if the fire identifier distinguishes smoke.

7. is ready to control the observation vehicle from anyplace to screen his/her home.

The point of this paper is to give a short outline of IoTbased Smart Home Environments with an emphasis on their empowering advancements, application zones, structures and designs. My objective isn't to give a point by point explanation of each subject, yet to give the peruser the fundamental standards and a short diagram of each subject, just as the list of sources to be checked in the event that somebody wishes to develop on certain parts of the subject.

\subsection{Enabling Technologies for IoT}

The prevailing improvements in statistics and correspondence advancements (ICT) diagnosed with pc structures, implanted frameworks and guy-made brainpower have made the vision of smart home simply potential. So through enhancing conventional home Automation systems with new first-rate capacities, it's been achievable for sensible domestic circumstance to display one-of-a-kind types of guy-made brainpower. Savvy domestic innovation is the consolidation of innovation and administrations thru domestic structures management for a advanced existence great. The empowering advances for IoT incorporate; Radio Frequency identity (RFID), internet Protocol (IP), digital Product Code (EPC), Barcode, wireless constancy (wireless), Bluetooth, ZigBee, close to Filed verbal exchange (NFC), Actuators, wireless Sensor Networks (WSN) and synthetic Intelligence (AI). For extra element study this writing [20]. 


\subsection{Application Areas of SHAS}

The Internet of things gives an adaptable and versatile stage that can bolster a wide range of uses. Its ubiquity has prompted an assortment of utilizations, including brilliant homes among others. The principle Smart Home Automation System (SHAS) application territory is ecological control with the customary help types lighting/day lighting and Heating, Ventilation and Air molding (HVAC) frameworks, observing and control, wellbeing and security, telehealth care, vitality sparing, natural control, and data get to [22]. There are various types of savvy homes application region; Smart homes for security, Smart homes for eldercare, Smart homes for human services, Smart homes for childcare, Smart homes for vitality proficiency and Smart homes for better life (music, diversion and so on.).

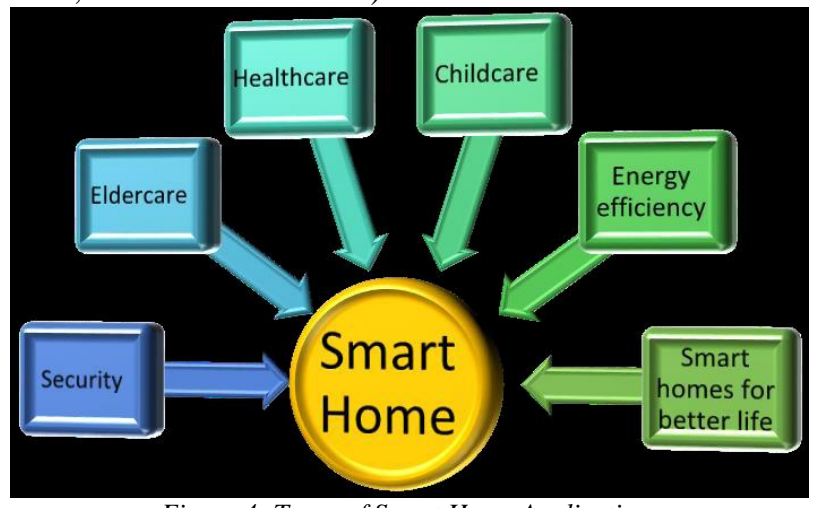

Figure 4: Types of Smart Home Applications

\section{3. $\quad$ Structure}

A smart home may be defined via a residence that is equipped with clever items; a home community makes it possible to move records between items and a residential gateway to attach the clever domestic to the outdoor net world. Clever items make it feasible to have interaction with inhabitants or to study them. Technically, home Automation system includes 5 building blocks.

\subsection{Devices under Control}

These devices consist of all additives, consisting of domestic home equipment or client electronics, which are related to and managed via the home automation device. Exclusive sorts of connecting technology inclusive of WLAN-, Bluetooth-, Z-Wave-interfaces, and many others. Are used for direct connectivity to the manipulate community.

\subsection{Sensors and Actuators}

Sensors may be study and watch, hear within the domestic system. There are sensors for a broad collection of employments, for example, estimating temperature, moistness, light, fluid, and fuel and figuring out development or commotion. Actuators are the strategies for the way the eager system can in all fact get matters carried out in fact. There are mechanical actuators, as an instance, siphons and electrical engines or digital actuators, for example, electric powered switches. The IoT gadgets geared up with sensors will cross about as government and the ones implanted with actuators will move about as entertainers. A system with each the sensors and actuator will see and carry out.

\subsection{Architecture}

In literature, the authors present a layer architecture model of smart home control system based on Internet of Things which includes Perception Layer, Network Layer and Application Layer.
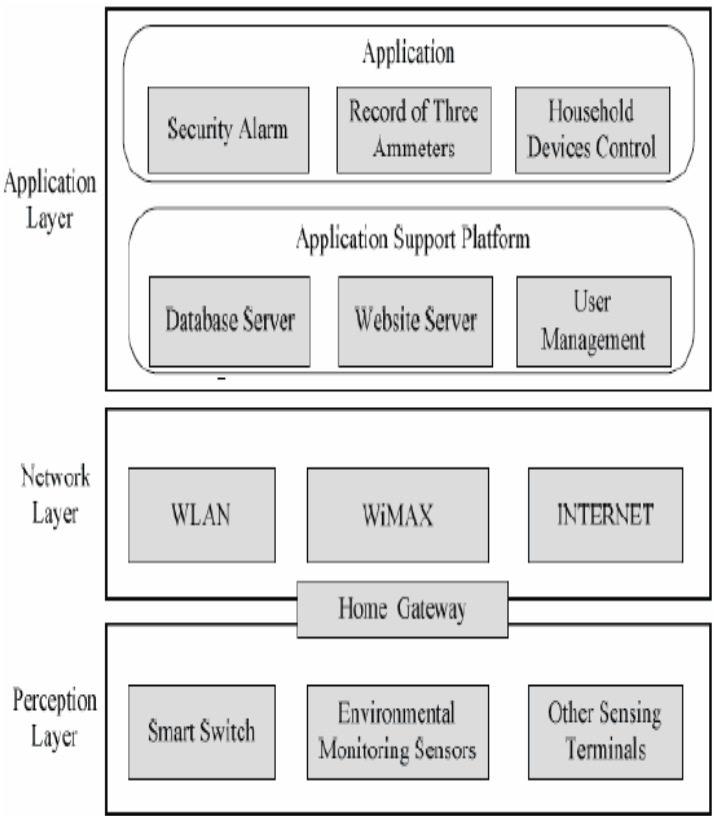

Figure : Layer architecture model of IoT-based smart home control system [27]

\section{PROBLEM FORMULATION WITH SECURITY RISK ASSESSMENT}

Within the accompanying areas we will play out a security danger appraisal and trouble formula for the IoT-primarily based fantastic home using the OCTAVE Allegro technique Both the approach and the IoT-based totally savvy domestic are as of now portrayed in the past sections. Basic records advantages for the savvy home can be prominent, alongside its vulnerabilities and capacity risks. At that factor an association to slight these dangers will be proposed. Earlier than we begin to practice the approaches of the security hazard evaluation system step by step, we want first to signify the safety chance appraisal itself simply as all phrases that we are going to use via the strategies of main the safety danger appraisal simply to make it honest. The goal of a hazard appraisal is to recognize the contemporary framework and condition, to recognize dangers and their consequences through investigation of the records collected. The incentive at the back of a security chance appraisal is to make bigger the coverage of class, respectability and accessibility by using giving guidelines without influencing the usefulness and simplicity of use. Here come some meanings of those terms that we are going to use through our safety risk appraisal procedure inside the device's worksheets inside the writing "presenting octave allegro: improving the statistics security threat evaluation technique".

- Asset - An asset of significant well worth. It might be manner, innovation, physical item or an man or woman.

- Facts Asset: it's miles extensive information for an affiliation that may be conveyed through individuals, put 
away in bodily Medias or moved and dealt with electronically.

- Facts useful resource compartment - The holder of the statistics aid is in which the records lives. Holders can be specialised (software programs, durable items, servers and systems), bodily (on papers, compact disc, dvd) or individuals (who thinks about the statistics). - critical facts resource: it is most giant aid that makes a big harm an association if its safety conditions are undermined.

- Hazard - The functionality of an event that might harm a advantage or good deal it. It's far created when a hazard entertainer abuses weak spot.

- Effect - The massive or impalpable impact of a threat being completed on a gain.

- Risk - its miles a mix of threat and effect. A threat is the threat of enduring harm or misfortune and is constructed from an occasion, a end result and vulnerability.

- Mitigation- The hobby of lessening the seriousness of risks or reducing the association's creation to the hazard through utilizing various measures

4.1 Confidentiality: making certain that lone accredited people (or frameworks) technique a records resource.

4.2 Integrity: making certain that a information resource remains within the proposed circumstance and for the meant purposes. It ensures that the facts are reliable and specific.

4.3 Availability: ensuring that the records aid stays open to permitted people.

The proposed research could be linked to reviewing statistics safety dangers in IoT-based totally great houses. This assessment journey examines the facts security threats in interfacing amazing devices to each other and to the internet even as organizing a savvy domestic to be able to make clients cautious approximately the security risks that might possibly abuse, improve security and supply proposition.

\section{MODEL DESIGN}

A essential part of the mission become scheming a version for simulation, checking out and studying effects. AutoCAD was utilized in designing the separate parts of the house version. The layout changed into given as an enter to a laser reduce machine that reduce cardboard to the numerous components of the design. After that, the model turned into assembled to shape the room model supplied in the beneath figures.

- The parts that were applied within the domestic Automation Simulation are:

- Two RaspberryPi 2 version B: The principle preparing and controlling unit of the framework. One turned into utilized for the room version and the alternative for the reconnaissance automobile.

- Servo Motor: It is going approximately as the entryway lock.

Infrared (IR) sensor: shows the existing circumstance of the front entryway, both opened or shut.

- Net digital camera: Acts as a reconnaissance digital camera for the room gushing snap shots of that room that are organized by the RaspberryPi. It makes use of OpenCV's picture preparing to have the choice to differentiate questions inside the room.
- Smoke Detector: It identifies hearth, making sure the health of the house.

- H-Bridges: every H-Bridge controls engines, are utilized to control the 4 DC engines of the vehicle.

- Wi-fi Dongle: connected to the Raspberry Pi via USB port to permit its connection to far off web in place of making use of Ethernet link.

\section{PROPOSED RESEARCH METHODOLOGY}

The foremost component that ought to be taken into consideration within the wake of choosing this subject became the framework design. Through what approach will the machines and devices communicate with an internet/portable software, get orders and send their repute? Would it not be a good concept for it to be a server consumer conference, in which there may be a right away affiliation between the gadgets and the application used by the consumer? Via what technique will an instantaneous affiliation be built up over the web? Or on the other hand need to the client and server bring through a medium channel that the messages must be handed on earlier than arriving at the consumer or the server? Those inquiries had brought about investigating various methodologies and administrations. Raspberry Pi changed into picked because the essential manipulate unit, to interface numerous sensors and gadgets together and to the web. The Raspberry Pi is a minor computer that has forty GPIO pins and in shape for being modified, so it become picked for its straightforwardness and capacities. The following take a look at, in the wake of figuring out how to accomplice numerous gadgets collectively and to the internet was sincerely controlling them over the net via an internet/flexible utility assembled utilizing Ionic Framework. Diverse ways and administrations were investigated remembering placing-up port sending for the transfer. Setting-up port sending will trade the con-figuration of the switch and uncover one port of the private neighborhood location network (LAN) to the general populace, that's regarded as a security defenselessness that must be overseen carefully. A portion of the administrations that have been investigated and considered to allow the correspondence of the web/versatile software with the Raspberry Pi associating various devices are Weaved, Pusher and AWS cellular Hub. Nonetheless, the need of ongoing cooperation and PubNub's distribute/buy in model, it became picked as the most first-class arrangement.

For you to have the choice to respond to the exploration addresses expressed above, there may be a want to pick out a appropriate studies gadget. The system acquired or advocated for this examination assignment (ace proposition) can be OCTAVE Allegro (OA) [44]. The OCTAVE Allegro methodology is intended to create strength in consequences, allowing exhaustive risk evaluation, concentrating totally on information sources. The method investigations how the records is utilized by the clients or frameworks. In addition, it facilities across the area where the records lives and on how it is presented to risks. Other basic assets can be distinguished and surveyed by means of locating the association among them and the records resource. OCTAVE Allegro gives route, worksheets and surveys for leading the chance appraisal. 

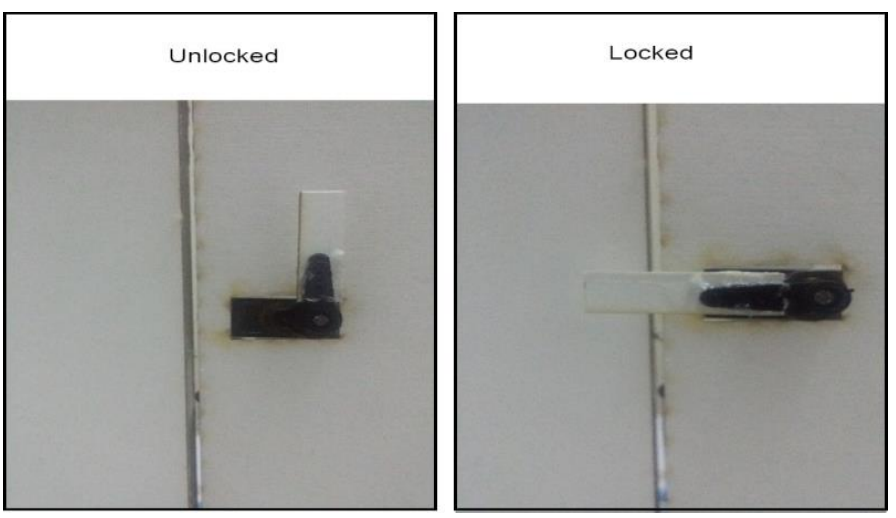

Figure Illustrates the submit/subscribe version provided by means of PubNub1.

Upon receiving the JSON string, the Raspberry Pi take the movement designated by way of that string. This lets in full manipulate and monitoring of all devices connected to the Raspberry Pi by means of the user, as illustrated within the preceding discern. The steps of the OCTAVE Allegro:

1) set up chance measurement criteria

2) broaden records Asset Profile

Three) identify information Asset containers

4) discover areas of problem

5) discover danger situations

6) become aware of risks

7) analyze dangers

8) pick Mitigation method

OCTAVE Allegro is satisfactory appropriate to answering the research problems compared with other protection hazard evaluation methodologies that were considered. It includes eight steps which might be organized into 4 levels and those steps can without difficulty be mapped to remedy my studies troubles. With the assist of worksheets provided through the technique can we seize the outputs from each step in the risk evaluation and use them to input into the following step which follows. In this way it allows us to preserve non-stop awareness on the asset step by step all through the procedure of danger evaluation and explore problematic conditions extra effortlessly.

\section{USE CASES AND RESULTS}

Both, the Raspberry Pi and the mobile application, are senders and receivers of data. This applies as both publish and subscribe at the same time to the same PubNub channel. This allowed bidirectional communication for data exchange. Accordingly, multiple use cases were implemented and studied for result analysis. The LEDs, connected to the Raspberry Pi through the GPIO pins and embedded in the model, were controlled through a mobile application developed with Ionic. A "Light" tab was created in the application which has a button that toggles between"ON" and "OFF" when clicked. The default value of the button shows the current status of the LED lights. If the button shows the value "OFF", this means that the LEDs are currently turned off. However, when the button is clicked, the value toggles to "ON", sending a JSON string holding a value of 1 for "led" attribute, 'led': 1 , to the channel the application publishes to, through PubNub and the "publish key". The JSON string received by this channel is then sent to its subscribers, the Raspberry Pi in this project's case. The Raspberry Pi then receives the JSON string, checking the "led" attribute value and turns the LEDs accordingly. The mobile application is also a subscriber to the same channel, in which it can check the last status of the LEDs published on PubNub channel and shows either "ON" or "OFF" as a default value of the button in the application. A 180 degrees servo motor, acting as the door lock of the modeled room, was connected to the Raspberry Pi through the GPIO pins. It was glued to the model's door along with a glued cardboard tip to lengthen the servo's horn. A "Lock" tab was created in the application which has a button that toggles between "LOCK" and "UNLOCK" when clicked. The default value of the button shows the action that will be taken by the servo lock, changing its angle, when the button is pressed. If the button shows the value "UNLOCK", this means that the servo motor will be adjusted to 0 degree, unlocking the door. The button's value toggles to "LOCK", When the button is clicked, sending a JSON string holding a value of 0 for "lock" attribute, 'lock':0, to the channel the application publishes to and the "publish key" provided by PubNub. The JSON string received by this channel is then sent to the Raspberry Pi, which receives the JSON string, checking the "lock" attribute value and sets the servo motor's angle to 0 degrees, unlocking the door.

A web camera was associated with the Raspberry $\mathrm{Pi}$ through a USB port for the room model observation. A straightforward movement identification calculation was composed and run utilizing OpenCV library to distinguish if the room is involved or not. The calculation identifies significant changes in the room condition as it has the default room condition foundation. On the off chance that a significant contrast distinguished between the foundations the calculation has and the ongoing caught picture, at that point the room is involved, else, it isn't. In the event that the room is involved, a JSON string holding the estimation of 1 for "occ" characteristic, 'occ': 1 , is distributed by the Raspberry Pi, to PubNub channel. The JSON string is then sent by that channel to its endorsers, the versatile application for this situation. At the point when the JSON string is gotten by the portable application, the "occ" quality worth is checked and a message is shown as needs be, if the room is involved, the message shows "The room is involved", if not, the message shows "The room isn't involved". This permits the client to consistently screen his/her room guaranteeing its security. The Raspberry Pi of the car was a publisher and a subscriber to the same channel, the other Raspberry Pi connected to the room model publish and sub- scribe to. This makes both Raspberry Pis able to receive the same messages, keeping the whole system synchronous. A camera module was connected to the Raspberry Pi of the car, which live streams to a YouTube channel, so that the user has an eye inside the house while he/she is away. The YouTube channel link was provided to the mobile application so that the user can control and monitor his/her home in one place. The mo- bile application has a tab that contains four control buttons that move the car forward, backward, left and right, along with the live video stream through YouTube, so that the user can see what he/she is controlling, and where to direct the car. The car control is done through sending JSON strings over PubNub. If the "Forward" key is pressed and 
held on the mobile application, a JSON string message, 'car':forward, is sent to the Raspberry Pi through PubNub. Accordingly, the car starts and keeps moving forward until the "Forward" key is released, which sends another JSON string from the mobile application to the Raspberry Pi over Pub Nub, 'car':stop, stopping the car and mak- ing it ready to receive another direction command the same way as forward command. The surveillance car can be used anytime to keep an eye of the user inside the house. If a security or safety breach is detected through the IR sensor on the door or the fire detector for example, the surveillance car can be a moving eye monitoring the house. It can check lively what is going on exactly, so that the user can take decisions accordingly and effectively. Results show that everything worked as anticipated, although some high pings in the network may cause minor occasional lag in the connection and data exchange, however, there was no major lag detected affecting the model control and testing. The proposed access monitoring and control mechanism at home is implemented using Raspberry Pi 3 which has 4x ARM Cortex-A53 processor operating at $1.2 \mathrm{GHz}$, Broadcom VideoCore IV graphics processor, 1GB LPDDR2 $(900 \mathrm{MHz})$ built in RAM, one 10/100 Mbps Ethernet port, $2.4 \mathrm{GHz} 802.11 \mathrm{n}$ built in wireless adapter and a 32GB class 10 micro Secure Digital (SD) Card as the hard disk storage. At the access point Arduino Uno microcontroller with ATmega328P IC is used to gather data. Arduino Uno module has fourteen digital input/output pins (6 of which can be used as Pulse Width Modulation (PWM) outputs), six analog inputs, a USB connector port, a $16 \mathrm{MHz}$ ceramic resonator, a power jack, an In-Circuit Serial Programming (ICSP) header, and a reset button. Arduino is flexible and offers a variety of digital and analog pins, it can be connected to a PC using USB, and it can run in standalone mode or as an interface connected to a PC. Arduino is cost effective and is an opensource project backed up by a strong online community.

A Light Dependent Resistor (LDR) is used to measure the intensity of light at various parts in the apartment. LDR uses photo-resistor with variable resistance which changes with the intensity of light. The resistance decreases with the increase in intensity of light on the sensor surface. Piezoelectric sensors are used to identify when the apartment keys are placed inside the bowl. Piezoelectric sensor uses the piezoelectric effect which converts between electrical and mechanical forms. In Piezoelectric sensor, the acoustical pressure is converted into electrical voltage which can be measured using the Arduino Uno module. Acoustic pressure is generated when the user places his keys into the bowl. Figure 4.1 (a) and (b) shows the deployment of piezoelectric sensor in the key bowl.

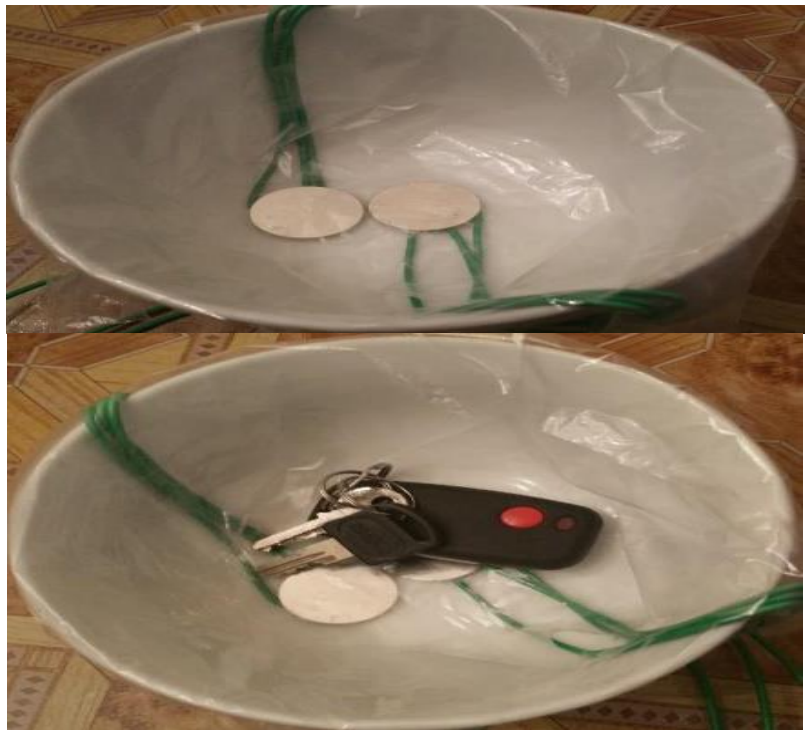

Fig: (a) Shows Piezoelectric sensor deployed in the key bowl. (b) Shows keys placed inside the key bowl.

The communication between the microcontroller and the Pi is wireless. Wireless communication technology is easy to install and reduces system cost. We considered various wireless technologies like Wi-Fi and Bluetooth. Wi-Fi was discarded because of its high power consumption and high cost, while Bluetooth was discarded because of high power consumption, limited range and security issues [20]. The proposed system is implemented using ZigBee technology based on the IEEE 802.15.4 standard with a communication range varying from $10-100 \mathrm{~m}$. ZigBee allows large-scale network configurations and utilizes low power radio with a data-rate capability of $250 \mathrm{~kb} / \mathrm{s}$. These features makes ZigBee the ideal communication technology in smart home networks. Moreover, many secure communication techniques with ZigBee [141] - [143] were suggested and successfully implemented. This makes ZigBee a comparatively secure wireless communication technology.

The plan and location of the sensor deployments in the apartment during the behaviour Four boards are designed to obtain logical sensing parameters. Board I is deployed near the primary access point. It consist of wires to proximity, motion and contact sensors to obtain various logical sensing parameters. The board is connected to the Arduino Uno module which supplies the power to the sensors. The Arduino Uno microcontroller is then connected to a ZigBee communication module. Figure 4.4 shows the board I and microcontroller deployment at Primary Access Point and Figure 4.5 shows board one installation at the primary access point. Board II is deployed near the secondary access point. It is connected to two contact sensors, a motion and proximity sensors. Each of the contact sensors are connected to window and balcony door. The motion and proximity sensors are deployed near the window to identify user movements before the window is opened. Board II also has provisions to be connected to Board IV which is connected to the force sensors in the bed. Similar to board I, board II is also connected to Arduino Uno microcontroller which powers the board and communicates the sensor values to the Pi using ZigBee module. Figure 4.6 shows board two installation at 
secondary access points and Figure 4.7 shows board II and microcontroller deployments at secondary access point.

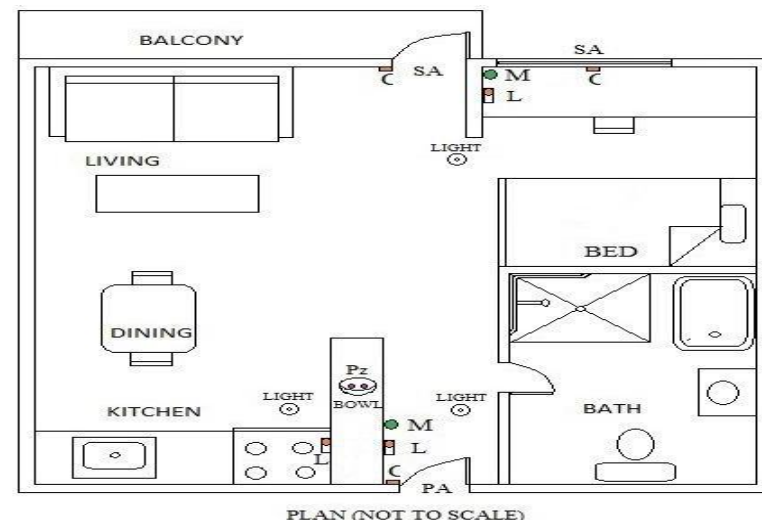

C - CONTACT SENSOR
- M-MOTION SENSOR

I I - LIGHT SENSOR

- Pz - PIEZOELECTRIC SENSOF PA - PRIMARY ACCESS POINT

SA - SECONDARY ACCESS POINI

Figure 4.3. Shows the plan and location of the sensor deployments in the apartment during the logical sensing.

Board III is deployed in the kitchen, it is connected to MQ 9 carbon monoxide sensor, DHT 11 temperature and humidity sensor. Board III is designed to measure the carbon monoxide, temperature and humidity levels in the area to detect fire. Similar to board I and II board III relies on Arduino Uno module for power and ZigBee module for communication.

Figure board three installation in the kitchen. Board IV is deployed near the bed it is connected to two circular 0.5 inch diameter force sensing resistors. Both force sensors are deployed underneath the mattress. Both force sensors has a full scale measurement accuracy of $\pm 5 \%$. Moreover, force sensors used in the experiment are readily available, cost effective, flexible and provides un-obstructive force measurements. Light sensors were integrated into boards I, II and III to measure the intensity of light near the primary access point and inside the apartment. to ZigBee communication modules to send sensor data to the Pi.

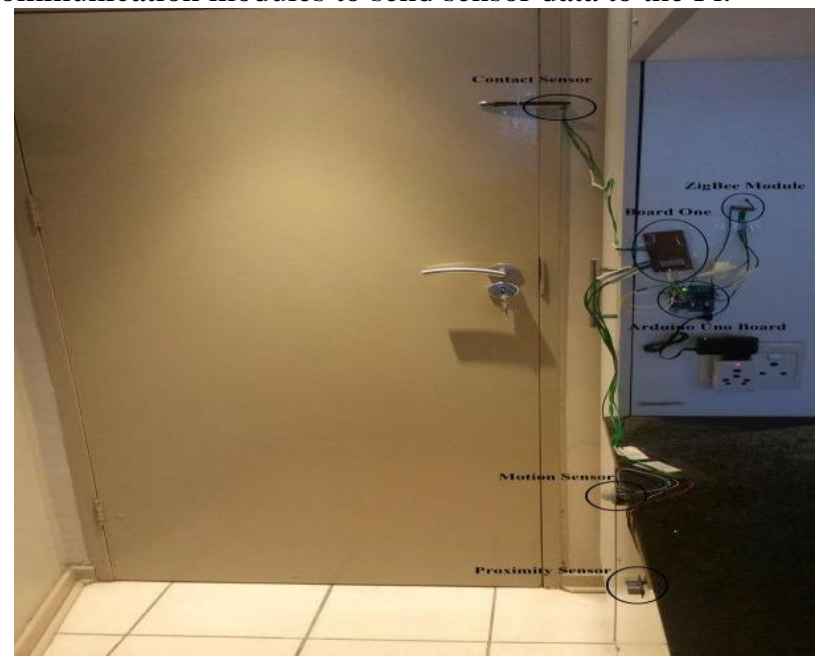

Figure 4.4. Shows Sensors, board and microcontroller deployment at Primary Access Point.

The power to each of the Arduino Uno boards is routed through a power bank. The power banks needs to provide a
9V-1000mA DC output to the Arduino boards. Any reliable power bank which has $5000 \mathrm{mAh}$ or higher capacity with $5 \mathrm{~V}$ $1000 \mathrm{~mA}$ USB power output would be enough to provide the power backup. The $5 \mathrm{~V}$ output power from the power bank is boosted to $9 \mathrm{~V}$ when connected to the Arduino board through a USB to $2.1 \mathrm{~mm}$ DC 9V Booster Cable.

The force bank is connected to the loft's $230 \mathrm{~V}-50 \mathrm{~Hz}$ AC power gracefully through a $5 \mathrm{~V}-1000 \mathrm{~mA} \mathrm{AC}$ to $\mathrm{DC}$ connector. In any event, when the ability to the loft is cutoff the Arduino Uno sheets and the sensors are as yet dynamic and will have the option to distinguish interruption endeavors by drawing power from the force bank. Figure 4.8 shows the altered force source. Contrasted with AA or AAA batteries power banks offers dependable and solid force flexibly after some time without substitution.

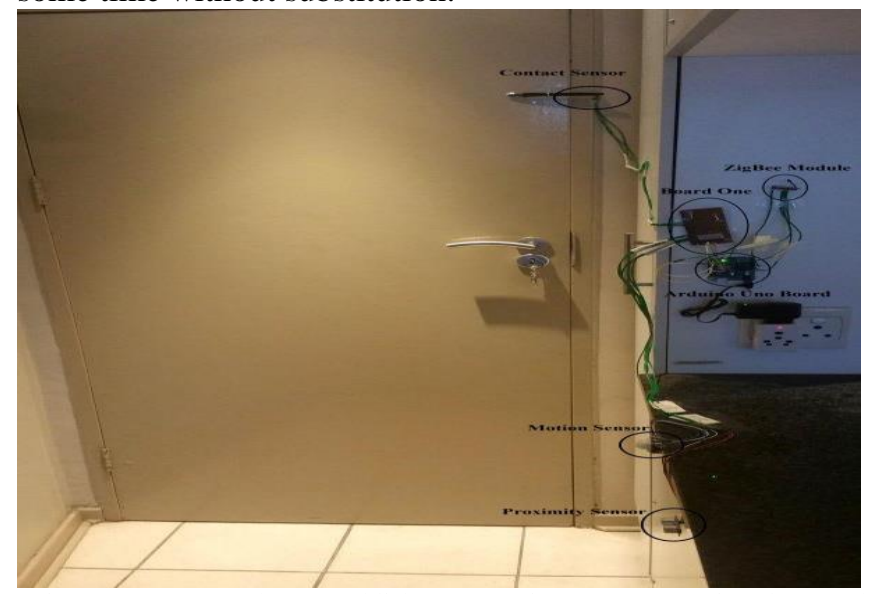

Figure 4.5. (a) Board one establishment at Primary Access Point. (b) Board one conveyed at essential passage with featured light sensor used by conduct forecast calculation.

All the ZigBee correspondence is executed utilizing ZigBee Series 1 module. ZigBee module at the $\mathrm{Pi}$ is designed as the ZigBee Coordinator (ZC) while the modules joined to the microcontrollers is arranged as the ZigBee End Device (ZED). The information pace of all the ZigBee modules are set at 9600 bits for every second (bps).

Figure 4.5. (a) Board one establishment at Primary Access Point. (b) Board one conveyed at essential passage with featured light sensor used by conduct forecast calculation. \#include <SmartHomeBILAL.h>

\#include <FlipMqtt.h>

SmartHomeBILAL fsh;

FlipMqtt m;

char* temp_topic ="TempratureHome";

char* hum_topic ="Home/murmur";

char* ldr_topic $=$ " Home/light";

char* switch_topic="Home/switch";

char* ssid="ssid";

char* pwd="PASSWD";

int temp, murmur, ldr;

char* $\mathrm{s}=\mathrm{NULL}$;

void arrangement()

\{

m.mqttSub(switch_topic);

m.mqttBegin(ssid,pwd);

fsh.relayOff(); 
void circle()

$\{\mathrm{s}=\mathrm{m}$.GetSubValue(switch_topic);

on the off chance that $(\operatorname{strcmp}(s, " 1 ")==0)\{$

fsh.relayOn();

else \{

fsh.relayOff();

temp $=$ fsh.readTempC();

m.mqttPub(temp_topic,temp);

murmur $=$ fsh.readHum () ;

m.mqttPub(hum_topic,hum);

$\mathrm{ldr}=$ fsh.readLdr();

m.mqttPub(ldr_topic,ldr);

\}

I* User can likewise characterize the vehicle for getting cautions and notices. The various mediums can be email, instant message, and internet based life. Client can pick any one or all. Following python content sends email alert if light power in higher. */

import FlipUtilities as Flip

import FlipMQTT as mq

import time

import getpass

$\mathrm{mqtt}=\mathrm{mq} \cdot$ FlipMQTT ()

mqtt.sub_topic("Home/light")

mqtt.infy()

administration $=$ 'gmail'

Flip.selectService(service)

username = "username"

PASSWD = "PASSWD"

to $=$ "to-email-address"

frm $=$ "from-email-address"

Flip.emailCredentials(username, PASSWD)

print "Signed in effectively!"

received_data $=[]$

check_timestamp $=$ ' 0 '

while True:

received_data $=$ mqtt.sub_value $($ "Home/light")

whenever got information != None and got data[1]!=

check timestamp:

print received_data

in the event that (received_data[0] >= "500") :

check timestamp = got data[1]

subject $=$ "Sensor Values"

message $=$ "Light Intensity is "+

received_data[0]+" Time:"+received_data[1]+""+"

Killing Room Light."+""

Flip.sendMessage(to, frm, subject, message)

print "message sent Successfully" .sleep(10)

Flip.closeEmail()

The proposed framework is useful in checking and controlling brilliant home condition. Utilizing this framework air quality can be constantly observed in home and alarms can be sent to client about wellbeing dangers assuming any. Proposed framework additionally improves security. Client can screen each action in home and can control windows and entryways. This framework likewise guarantees better use of vitality and assets through shrewd lighting, savvy machines and keen cooling framework. Fig. 8 presentations email notice got at client's enrolled email account as the higher light power recognized in the room and accordingly room lights were consequently killed by the framework.

\section{CONCLUSION}

Web of Things has various applications in different zones. IoT has been starting at now planned for mechanical WSN. It has been made for Smart Homes System. There are a couple of issues found in IoT and Smart Homes. New advances could help with constraining some of them. This paper presents the issues and challenges that could come. The theory targets were to present the subject of Internet of Things (IoT) and its application to make sharp homes to give getting, comfort and to improve the individual satisfaction. Bringing IoT advancement to our home outcomes in new security challenges, in this manner IoT-based awe inspiring homes require extreme security basics.

These moved improvements offer the two prospects and dangers, an IoT-based Smart Home is especially powerless against various security dangers both from inside and outside the home, if security in a shrewd home or astonishing gadget was undercut, and the client's security, solitary data and regardless, success of the occupants will be at risk. Along these lines, sensible assessments must be taken to make the watchful home dynamically secure and appropriate to live in. In any case, we should know precisely what we are trying to ensure about and why before picking unequivocal blueprints. Home Automation is one of the critical usages of IoT. It gives less complex and entertainment living to every person. In this endeavor, a system for working up an IoT programming based canny home computerization structure was completed and attempted through the made model. It bases on prosperity and security perspective of home computerization through using a bit of the progressing open advances. The logical investigations showed satisfying outcomes with uncommon and satisfactory leeway, which maybe caused as a clarification of the utilization of $4 \mathrm{G}$ affiliation.

\section{REFERENCES}

[1] Malche, Timothy, and PritiMaheshwary. "Internet of Things (IoT) for building smart home system." 2017 International Conference on I-SMAC (IoT in Social, Mobile, Analytics and Cloud)(I-SMAC). IEEE, 2017.

[2] Gabhane, Mrs Jyotsna P., MsShradhaThakare, and Ms Monika Craig. "Smart Homes System Using Internet-of-Things: Issues, Solutions and Recent Research Directions." International Research Journal of Engineering and Technology (IRJET) 4, no. 5 (1965): 1969.

[3] Khan, Akram, et al. "Design of an IoT smart home system." 2018 15th Learning and Technology Conference (L\&T). IEEE, 2018

[4] Bhavna, Dr, and Neetu Sharma. "INTERNATIONAL JOURNAL OF ENGINEERING SCIENCES \& RESEARCH TECHNOLOGY SMART HOME AUTOMATION USING IOT

[5] Shouran, Zaied, Ahmad Ashari, and Tri Priyambodo. "Internet of Things (IoT) of Smart Home: Privacy and Security." International Journal of Computer Applications 182 (2019): 3-8.

[6] Tiwari, Umesh Kumar, and Priya Matta. "Efficient Smart-Home Architecture: An Application of Internet of Things." Available at SSRN 3350330 (2019

[7] Neuman, B. Clifford, and Theodore Ts'o. "Kerberos: An authentication service for computer networks." IEEE Communications magazine 32.9 (1994): 33-38.

[8] Lu, Jiakang, DagnachewBirru, and Kamin Whitehouse. "Using simple light sensors to achieve smart daylight harvesting." Proceedings of the 2nd ACM Workshop on Embedded Sensing Systems for Energy-Efficiency in Building. ACM, 2010. 
[9] Anitha, A. "Home security system using internet of things." IOP Conference Series: Materials Science and Engineering. Vol. 263. No. 4. IOP Publishing, 2017.

[10] Desai, Drushti, and HardikUpadhyay. "Security and privacy consideration for internet of things in smart home environments." International Journal of Engineering Research and Development 10.11 (2014): 73-83.

[11] Sharma, M. L., Sachin Kumar, and Nipun Mehta. "SMART HOME SYSTEM USING IOT." International Research Journal of Engineering and Technology 4.11 (2017): 1108-1112.

[12] Sharma, M. L., Sachin Kumar, and Nipun Mehta. "SMART HOME SYSTEM USING IOT." International Research Journal of Engineering and Technology 4.11 (2017): 1108-1112.

[13] Bourobou, S., \&Yoo, Y. (2015). User activity recognition in smart homes using pattern clustering applied to temporal ANN algorithm. Sensors, 15(5), 11953-11971.

[14] Hoque, Mohammad Asadul, and Chad Davidson. "Design and Implementation of an IoT-Based Smart Home Security System." International Journal of Networked and Distributed Computing 7.2 (2019): 85-92.

[15] Jena, K. K., Bhoi, S. K., Maharana, P. K., Das, P. R., \&Senapati, P. K. A Smart and Secure Home Automation System Using IoT.

[16] Luigi Atzori, Antonio Iera, and Giacomo Morabito. The internet of things: A survey. Computer Networks, 54(15):2787 - 2805, 2010.

[17] Nicola Bui and Michele Zorzi. Health care applications: A solution based on the internet of things. In Proceedings of the 4th International Symposium on Applied Sciences in Biomedical and Communication Technologies, ISABEL '11, pages 131:1- 131:5, New York, NY, USA, 2011. ACM.

[18] L. Coetzee and J. Eksteen. The internet of things - promise for the future? an introduction. In IST-Africa Conference Proceedings, 2011 pages 1-9, May 2011.

[19] L Heuser, Z Nochta, and NC Trunk. Ict shaping the world: A scientific view, 2008. 